\title{
Solving the riddles of multiverse from the position of energodynamics
}

\begin{abstract}
The article highlights the events that preceded the theoretical prediction and recognition of the existence of black holes. The properties of super massive objects, taken for black holes, and the arguments that served as the basis for awarding the Nobel Prize in this area are analyzed. Based on astrophysical data, it is concluded that the black hole hypothesis does not explain most of the observed phenomena. An energodynamic theory of strong gravity is proposed, which sheds new light on the cosmogony of the Universe as a whole.
\end{abstract}

Keywords: baryonic and non-baryonic matter, their circulation, laws of gravity, attraction and repulsion, evolution of the Universe
Volume 4 Issue 4 - 2020

\author{
VA Etkin \\ Advisor to the Vice-Rector for Science, Scientific Center, \\ Togliatti State University, Russia

\begin{abstract}
Correspondence: VA Etkin, Advisor to the Vice-Rector for Science, Scientific Center, Togliatti State University, Russia,
\end{abstract} \\ Email etkinv5@gmail.com \\ Received: December 20, 2020 | Published: December 30, \\ 2020
}

On October 6, 2020, the names of the Nobel Prize laureate in physics became known. ${ }^{1}$ They were Roger Penrose from Great Britain ("for the discovery that the formation of a black hole is a reliable prediction of general theory of relativity"), and Andrea Ghez from the USA and Reinhard Genzel from Germany ("for the discovery of a super massive compact object in the center of our Galaxy"). Thus, they all received an award, according to the Nobel Committee, for clarifying the "darkest secrets of the Universe", and not for confirming the existence and not for studying black holes.

It is interesting to briefly consider the path that led them to such a high award, and the specific achievements that made them considered worthy of it. I will start with Reinhard Gentzel and Andrea Gez, who organized in the early 1990s. Observation of the center of our Galaxy with the 8-meter telescope in Chile and the 10-meter telescope named after Keck at the European Southern Observatory in Hawaii. They studied the motion of stars around a supposed super massive black hole at the center of the Milky Way. The center of this galaxy is hidden from us by dust clouds, and it is impossible to see it in visible light. Therefore, it was necessary to go to the near infrared region. To obtain images with the maximum diffraction angular resolution available for telescopes, these groups first used speckle interferometry, and thenadaptive optics.

Speckle interferometry in astronomy is a method of ground-based optical observations based on the analysis of the fine structure of "instantaneous" images of space objects. ${ }^{2}$ It allows you to obtain high angular resolution in the presence of atmospheric image distortion. The fact is that in a real telescope, due to wave front distortions in the atmosphere, the "instantaneous" image of a point (the so-called speckle image with a shutter speed of $\approx 0.02 \mathrm{~s}$ ) breaks up into a set of diffraction spots with a size of $\sim 1$ "due to wave interference, received random phase delays in the atmosphere. As a result, for example, a close binary star looks like two identical pictures. To cut this, the photographs are averaged over many images (from tens to a million). In the 1970 s, the theory of speckle interferometry was created. That is, it was shown that speckles are chaotic interference fringes that can be weakened by accumulating a large number (up to 106) frame. This method has been used since 1972, first by recording an image on a photographic film, and now-using television photon counters. As a result, the existing telescopes managed to improve the effective resolution by an order of magnitude, bringing the angular resolution to 0.0001 ".
A fundamentally different method of cutting irregular distortions arising from the propagation of light in an inhomogeneous medium is adaptive optics. This method is based on the use of controlled optical elements to increase the resolution limit of observation devices. His idea was proposed by the Russian scientist VP Linnik in $1957,{ }^{3}$ but the possibility of creating such a system appeared only in the mid1990s in connection with the development of technologies and with the possibility of the most correct computer control and monitoring. The main tool for controlling the wavefront and correcting optical aberrations is a deformable mirror. Unimorphic mirrors are widely used. Such a mirror consists of a thin plate made of piezoelectric material, on which electrodes are in a special way. The plate is attached to a substrate, on the front surface of which an optical surface is formed. When a voltage is applied to the electrodes, the piezoelectric plate contracts or expands, this leads to bending of the optical surface of the mirror. The special spatial arrangement of the electrodes of several hundred such actuators makes it possible to change the shape 1664 times per second and to form complex surface reliefs in real time to compensate for dynamic aberrations. ${ }^{4}$

However, in astronomical applications for adaptive optics systems, a reference source is needed that would serve as a brightness standard for correcting distortions created by atmospheric turbulence, and it should be found at a sufficiently close angular distance from the studied region of the sky. At first, secret military developments were used for this purpose, serving to check satellites. Subsequently, "artificial stars" were created as such a source by exciting sodium atoms with a ground-based laser at an altitude of about 90-100 km above the Earth's surface. These systems allow simultaneous tracking of up to ten or more sources in a field of view with a diameter of 5-10 arc minutes.

The use of this technique allowed Reinhard Hentzel and Andrea Guez to obtain, because of many years of observations of the motion of stars around a single common center, convincing evidence of the existence in the center of our Galaxy of a certain "compact and supermassive object" with a mass of 4.6 million solar masses. Moreover, in size it does not exceed the solar system. It would be more correct to call this object not a BH, but a galactic nucleus ", since it may turn out to be some other object. Astronomers have built a map of our galaxy (Figure 1), which made it possible to decide the center of the Milky Way more accurately. It turned out that this center is found at 25.8 thousand light years from the Earth, that is, closer to the 
previously accepted value of 27.7 thousand light years, and the solar system moves around the galactic center at a speed of $227 \mathrm{~km}$ per second, i.e. faster than previously accepted $220 \mathrm{~km} / \mathrm{s}$. These results, obtained and agreed upon by the collaboration of many astronomical laboratories, are a significant contribution to the study of "the darkest mysteries of the Universe".

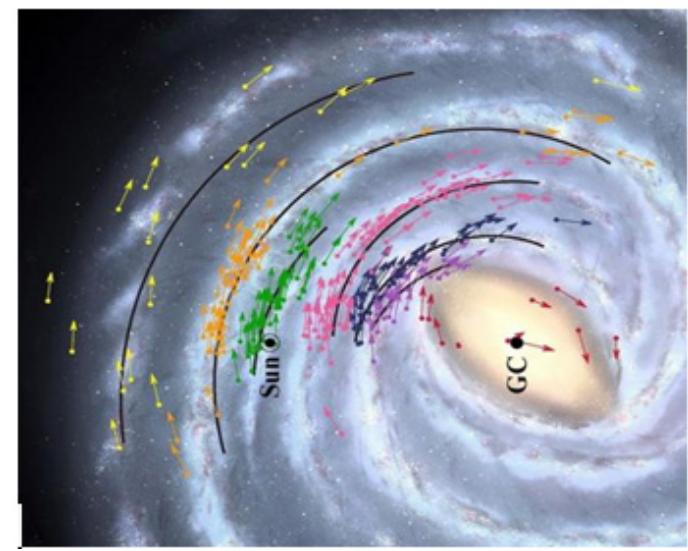

Figure I Map of the Milky Way galaxy.

The situation is much more complicated about Roger Penrose. He is the most famous and authoritative of the mathematicians working in the field of the mathematical theory of relativity and proved several general theorems about black holes (BHs), in which he substantiated the existence of an "event horizon" between the past and the future and the inevitability of the collapse of the BH under arbitrary first conditions. R. Penrose studied the structure of space-time around and inside black holes, resorting to rather elegant mathematical methods. The foundations of this method were formulated by him in his 1965 article "Gravitational collapse and space-time singularities" published in Phys. Rev. Lett., ${ }^{5}$ that is, only 10 years after the death of Einstein. In it, he substantiated that black holes are formed from a cosmological singularity, that is, states with infinite density and temperature, where all laws known to us cease to run. With the help of elegant mathematical models, he showed that the very existence of BHs is a direct consequence of the theory of relativity, although A. Einstein himself did not believe that objects that "devour" everything within their limits (and even light) exist at all. Thus, in his 1939 article, he even explains why "Schwarzschild singularities" do not exist in physical reality". ${ }^{6}$ Thus, he essentially renounces his own prediction of the existence of $\mathrm{BH}$. Therefore, some researchers consider R. Penrose even more consistent adherent of the theory of black holes than A. Einstein himself.

R. Penrose was the first to explain the origin of BHs by gravitational collapse, that is, by the catastrophically rapid compression of massive stars under the action of gravitational forces after exhausting their supply of thermonuclear fuel. From that time on, black holes began to be considered as a real, not a mathematical object.

Meanwhile, in the light of recent astronomical discoveries, the interpretation of the physical essence of BHs given by Penrose does not seem so unambiguous. To understand what we are talking about in general, let us name the most famous properties of "black holes" today:

a. The density of a $\mathrm{BH}$, defined as the ratio of its mass to the volume of the "Schwarzschild sphere" (on the surface of which the 2 nd cosmic speed is equal to the speed of light), are inversely proportional to the square of its radius. Therefore, in the Milky Way galaxy, it is close to the air density in the lower layers of the Earth's atmosphere and ranges from $0.06 \mathrm{~kg} / \mathrm{m}^{3}$ for the quasar OJ 287 to $1018 \mathrm{~kg} / \mathrm{m}^{3}$ for the smallest $\mathrm{BH}$, which exceeds the density of nuclei and neutron stars. Thus, singularity and collapse are only sufficient, but not yet necessary, conditions for the occurrence of $\mathrm{BH}$.

b. Black holes, by definition, have such a powerful gravity that even light cannot leave it. Therefore, they are completely opaque. Meanwhile, there are photographs where the stars found behind it are visible through a dark hole in the center of the galaxy (Figure 2). This means that far from all central regions of galaxies can be classified as $\mathrm{BHs}$, especially since in a huge number of cases an increased luminosity is seen at the centers of galaxies (Figure 3).

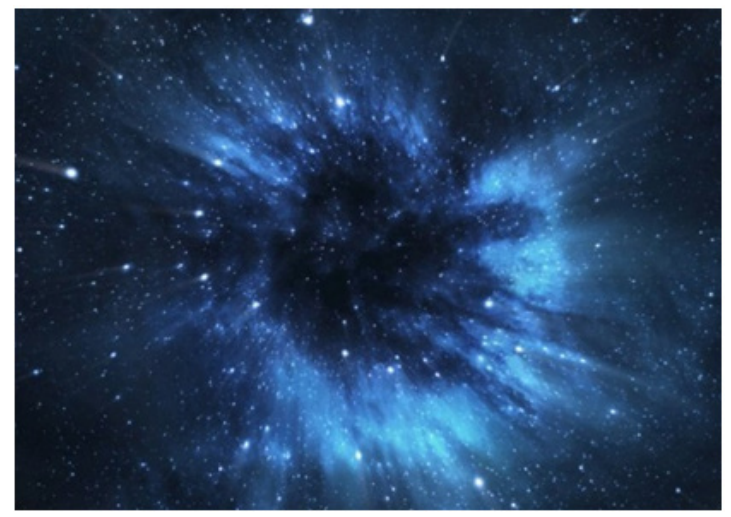

Figure 2 View of the stars through the "black hole".

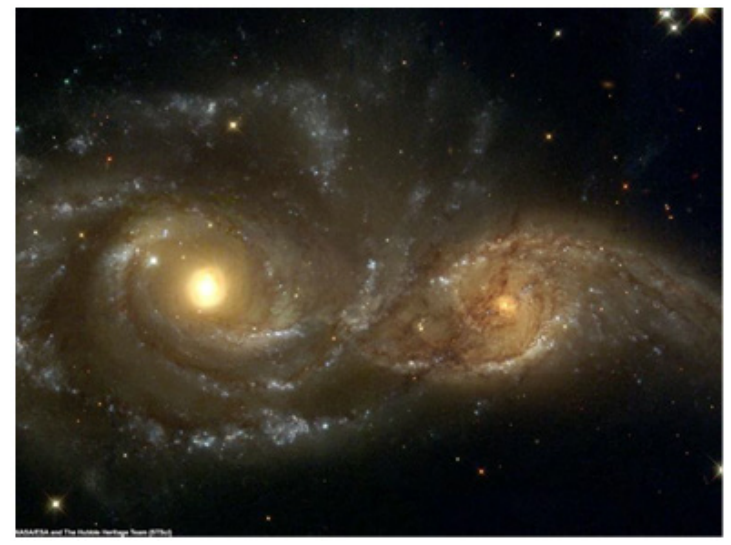

Figure 3 Zones of increased luminosity in the centers of galaxies.

c. It is assumed that the rays of light have a certain mass and move along the border of this sphere, without crossing or leaving these boundaries. However, this assumption, in principle, cannot be verified and therefore belongs (according to Popper) to the category of unscientific postulates.

d. According to Penrose, black holes are born at the site of the explosion of red giants, which occurs after the star depletes the "reserves" of thermonuclear fuel and its "collapse". Meanwhile, spectral analysis of stars born in the centers of galaxies reveals the presence of significantly more oxygen and other gases at the bottom than in the centers of distant galaxies, where there is much more iron. This shows that with age there is a "production" of heavy elements, and not their consumption. In addition, there are millions of stars in galaxies, which makes their simultaneous collapse unlikely.

e. Many spiral galaxies have been discovered, the central part of which ejects "jets" up to 250 million parsecs long, accompanied 
by powerful X-rays and even baryonic matter clumps (Figure 4). They are jets emanating from the central part of galaxies from elementary particles, gases, dust and even stars up to 2.5 times the mass of the Sun, along the normal to the axis of their rotation with a speed reaching six times the speed of light. This means that their denser part of galaxies becomes not a burial ground, but a "factory" of baryonic matter, thereby keeping the "universal mass balance".

f. It is known that galaxies can "swallow" each other along with their black holes. At the same time, they emit not only gravitational waves of space-time predicted by general relativity, but also ordinary acoustic (longitudinal) waves, perceived at distances of over 250 million light years. Their appearance is detected by the enhanced glow in the region of the shock front (Figure 5).

g. Astronomers very often see cases when stars in the accretion disk are thrown away from the center of the galaxy. This shows that along with the gravitational forces in the Universe, there are also gravitational "repulsion" forces. The presence of such forces is showed by the concentric structure of star clusters, when some of them are in the center, and the other at a certain distance between them (Figure 6), ${ }^{7}$ as well as keeping nebulae at a certain distance from each central cluster in the same figure.

h. According to celestial mechanics based on Newton's law, the rotation speed of the peripheral regions of spiral galaxies should decrease. However, correct measurements of this speed revealed its practical constancy. This discrepancy between the rotational curves of galaxies dictates the need to change not only Newton's law of gravitation, but also the entire theory of gravity.

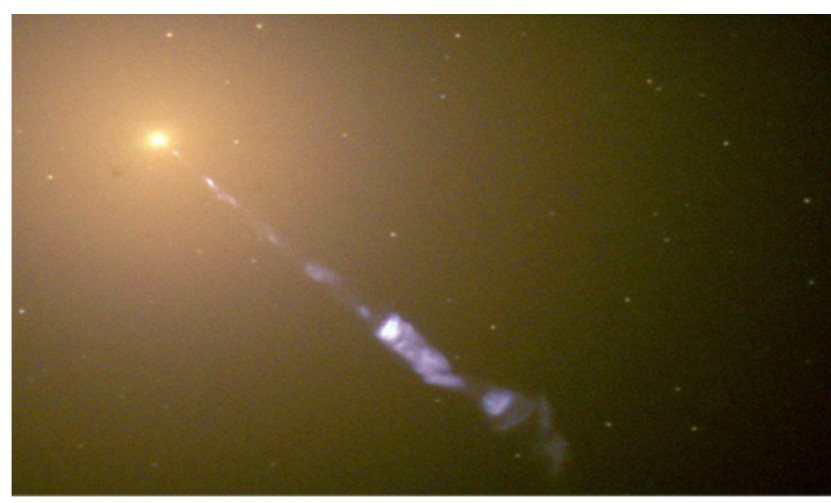

Figure 4 Jets emitted by spiral galaxies.

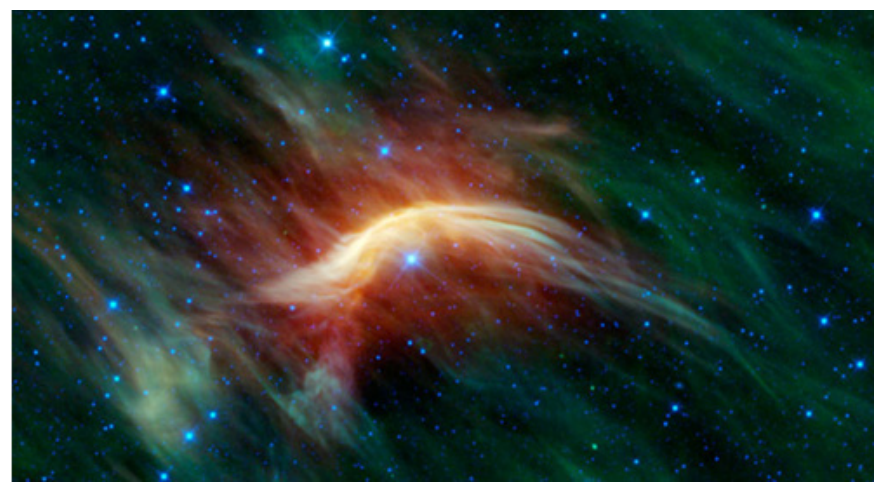

Figure 5 Shock waves from galaxy mergers.

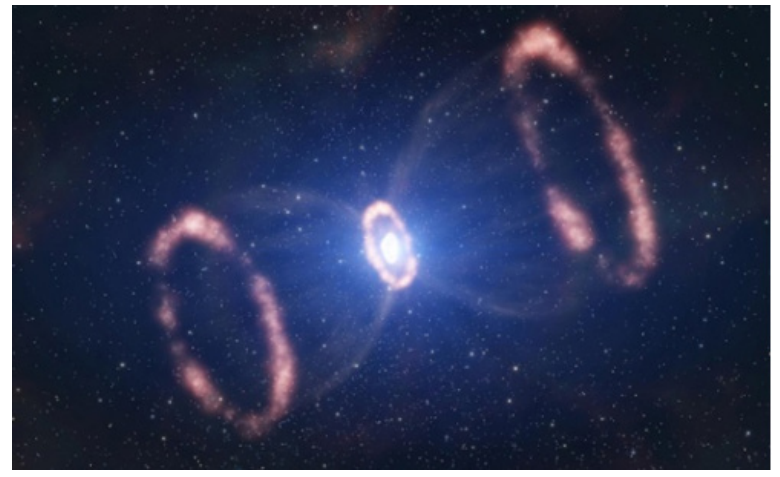

Figure 6 Ring structure of the galaxy and nebulae.

Approaching the theory of "black holes" from these positions, we must state that it does not give a satisfactory answer to any of the above questions. First, the question remains unanswered: where did these "reserves" of nuclear fuel come from and where is the source of baryonic matter, which is necessary to maintain its balance as the BH stars are "absorbed" by their "gravediggers". Such a source is also necessary to explain the observed phenomenon of the constancy of the density of baryonic matter during the "expansion" of the Universe. The physical vacuum (PV), which produces only "virtual" particles, is clearly not suitable for this purpose. Further, according to the "thermodynamics of BH", in which the surface of the "event horizon" is an analogue of entropy, the absolute temperature $\mathrm{T}$ of the $\mathrm{BH}$ should be nonzero, generating thermal radiation contrary to the definition of the BH, and its "evaporation". This discovery by S. Hawking testifies to the internal inconsistency of the BH theory.

All the above-mentioned inconsistencies force researchers to look for a way out of the framework of the "Standard Cosmological Model" based on general relativity. One of the attempts of this kind was undertaken by me within the framework of a unified theory of transfer and transformation of any forms of energy, called for short energy dynamics. ${ }^{8}$ Unlike general relativity, which replaced dynamics with kinematics, forces $\boldsymbol{F}$ with curvature of space, and physics with mathematics, energy dynamics gives a universal definition of force as a gradient of the corresponding form of energy $\mathrm{F}=\partial \mathrm{U} / \partial \mathrm{r}$. In this case, any force fields appear because of the inhomogeneous distribution in space of the material carrier of the corresponding form of energy. If general relativity is the dynamics of the gravitational interaction of the Universe by ten cosmological equations of Hilbert-Einstein in partial derivatives of the second order with hundreds of unknown variables, then energy dynamics is it in the form of one equation with two unknowns. Finally, this theory is based not on postulates, but on the astronomical discoveries of recent times mentioned above.

The most significant of these is the discovery that only $4-5 \%$ of the matter in the Universe is visible (observable). It is customary to call it "baryonic matter" because its structure has protons and neutrons. The rest of the matter is "hidden" (invisible). It is generally accepted that it consists of $22 \%$ of "dark matter", which forms a rotating "halo" around galaxies, and $74 \%$ of "dark" energy, which handles their accelerated recession (Figure 7). The structure of this "finer" matter does not lend itself to study by existing means, therefore we are forced to consider it a continuum. This corresponds to the division of matter in the Universe not into matter and field, but into structured (baryonic) and unstructured ("non-baryonic") parts. Non-baryonic matter has the only (gravitational) degree of freedom, and, like ether, fills all the space provided to it. Therefore, it is an indispensable part of any 
material system. This makes it the "primary" form of matter (presubstance), from which in the process of "condensation" (structure formation) all known forms of baryonic matter (elementary particles, nuclei, atoms, molecules, gases, dust clouds, small and large celestial bodies, galaxies, and their clusters). It is a small fraction of baryonic matter, the emission of which has an individual spectrum that decides the low temperature of the galactic medium $(2,73 \mathrm{~K})$.

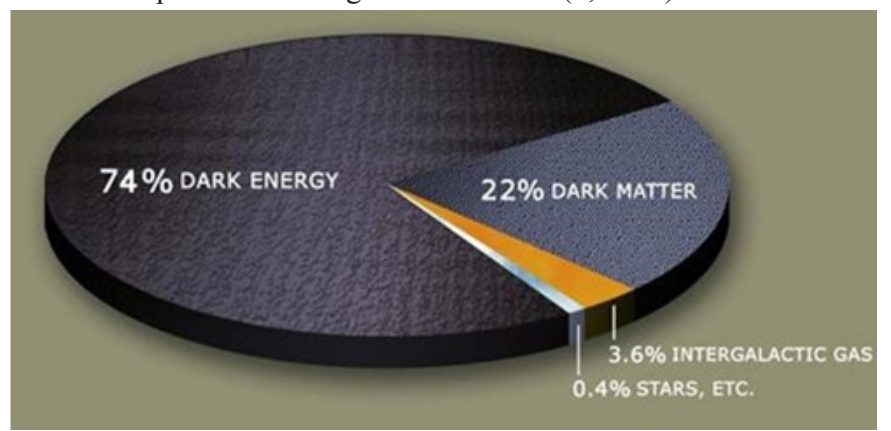

Figure 7 The balance of matter in the universe.

The existence of "voids" - cosmic voids of huge sizes (over a billion light years), free of baryonic matter, are confirmed by photographs (Figure 8). Energy dynamics explains them by the absence of conditions in the voids for condensation of non-baryonic matter. The density of non-baryonic matter in voids varies, according to modern data, from $\sim 10^{-27}$ to $\sim 10^{-34} \mathrm{~g} \mathrm{~cm}^{-3}$. Such in homogeneity makes it unstable and gives rise to self-oscillations of density in it. As proved in energy dynamics, the energy of these vibrations is $\varepsilon_{\mathrm{g}}=\mathrm{Mc}^{2}$ , that is, the value currently accepted as the total energy of matter. This energy makes non-baryonic matter the "fuel" of the stars, although it is only a fraction of the energy of non-baryonic matter, which is involved in oscillatory motion.

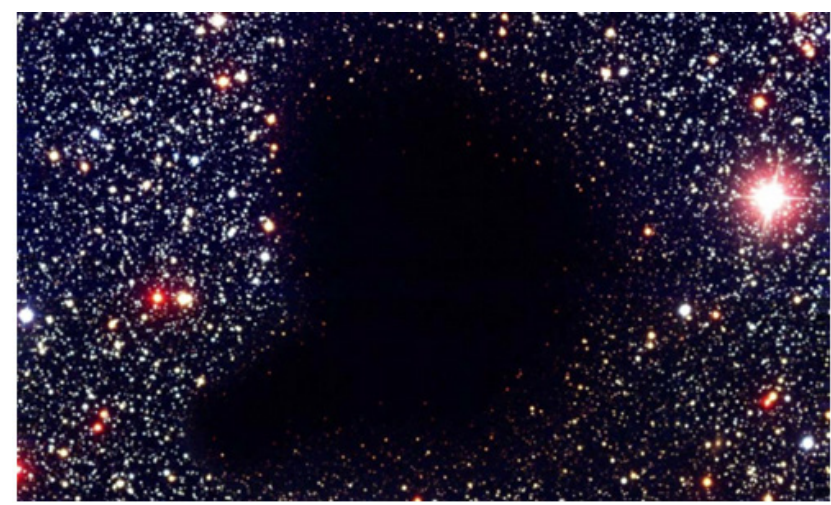

Figure 8 Void "Bootes".

Hence, under the assumption of the constancy of the speed of light $\mathrm{c}=$ const, the principle of equivalence of energy and mass of A. Einstein follows $\varepsilon_{\mathrm{g}}=\mathrm{Mc}^{2} .{ }^{8}$ This principle is sufficient to prove that the strength of the gravitational $\boldsymbol{X}_{g}$ and any other field is decided by the density gradients of the corresponding energy form $\varepsilon_{\mathrm{g}}=\partial \varepsilon_{\mathrm{g}} / \partial \mathrm{v}=\rho \mathrm{c}^{2}$, from which the law of gravity of the form follows directly:

$$
\mathrm{X}_{\mathrm{g}}=\rho \mathrm{g}=\mathrm{c}^{2} \nabla \rho,\left(\ddot{\mathrm{a}} \mathrm{M}^{-4}\right)
$$

The field (short-range) form of the law of gravity (1) is irreplaceable for continuous media, in which it is impossible to distinguish between "field-forming" and "test" bodies. It predicts the existence of strong gravity ( $\sim 10^{46}$ times greater than Newtonian), gravitational equilibrium $(\nabla \rho=0)$, the possibility of changing attraction $(\nabla \rho>0)$ by repulsion $(\nabla \rho<0)$ when the nature of the distribution of matter in space changes (sign change $\nabla \rho$ ) and the disappearance of gravity upon reaching the limit of compressibility $(\rho=\rho \max ) .{ }^{8}$ The enormous size of the condensation energy $M c^{2}$ reveals an energy source hundreds of times higher than the thermonuclear energy, which sheds new light on the origin of the super-powerful radiation of quasars. The author's rationale for the short-range law of strong gravity allowed the Israel Association of Inventors to issue me a diploma in 2018 for "the discovery of the phenomenon of gravitational repulsion" (№ 017-2018)

Considering the spatial in homogeneity of the density distribution, the bipolar form of the law of gravity (1) eliminates the contradiction between Newton's law of gravitation and the observed nature of the rotational curves of spiral galaxies, leading to the law of their density distribution $\rho$ to the form: ${ }^{9}$

$$
(\mathrm{v} / \mathrm{c})^{2}=-\mathrm{r} \nabla \rho / \rho
$$

According to this expression, the speed $v$ of the differential rotation of the galaxy at a distance $r$ from the center decreases towards the periphery, the slower the sharper the decrease in the relative gradient of its density $(\nabla \rho / \rho)$, and at a certain value it can remain unchanged, which is observed.

The prediction of the existence of gravitational repulsive forces in baryonic and non-baryonic matter makes it unnecessary to introduce added entities such as "dark matter" and even more "dark" (unknown) energy, which handles the accelerated expansion of the Universe. Nevertheless, the energy-dynamic theory of cosmogony built on this basis allows one to explain the above-mentioned observational data without going beyond the framework of classical physics.

Indeed, according to the energy-dynamic theory of strong gravity, ${ }^{10}$ based on the law (1), the compaction of non-baryonic matter $(\nabla \rho>$ 0 ), having spontaneously arisen in some region of the Universe, does not change its sign in the future. Thus, the densification of any region of the Universe, once started, does not stop until the appearance of a singularity (a state with infinite density and temperature), unless the growing internal pressure forces cause it to "burst" on the way to this state. Such an "explosion" is inevitable, since as the singularity is approached, the relative density gradients $\nabla \rho / \rho$ and the gravitational forces become weaker, while the internal pressure forces caused by thermonuclear reactions, on the contrary, increase. This phenomenon of violation of hydrostatic equilibrium should be called "explosion" not "supernova", but "super old" since it completes the evolution of the star. The local expansion going with the explosion ends in the so-called "big rupture" of all its structures of baryonic matter, including atoms. This "break" returns the substance to its original (non-baryonic) state. The disordered alternation of the processes of contraction and expansion of individual regions of the infinite Universe realizes the circulation of matter, which allows it to exist indefinitely, by passing the state of equilibrium.

The energodynamic theory of cosmogony describes the process of transformation of non-baryonic matter into baryonic matter just as "prosaically". The point is that longitudinal acoustic vibrations of non-baryonic matter, detected in outer space, ${ }^{11}$ cover the entire possible frequency range $0<v<\infty$. Moreover, due to the "all-pervading" nature of gravity, the radiation going with these oscillations is background. This makes non-baryonic matter invisible (“dark"). Only in its "condensation", accompanied by structurization (formation of baryonic matter with an individual radiation spectrum), does matter 
become "visible" (observable). This is recorded by telescopes as the birth of a "supernova".

However, the condensation process occurs only when a certain density of non-baryonic matter is reached. Until then, the nucleus of any galaxy, like voids free of baryonic matter, is generally invisible, although it has nothing to do with BH. Such a core is transparent, which is reflected in Figure 1. It can be detected only by the motion of baryonic matter, carried away by the flow of non-baryonic matter. However, as it becomes denser and the condensation process occurs, the core begins to emit "jets" and becomes more and more luminous. This glow stops only with the "aging" of the galaxy, when the galactic nucleus becomes more homogeneous, and the relative value of the pressure gradient $\nabla \rho / \rho$ and the associated accretion of baryonic and nonbaryonic matter weaken. When approaching this state, the jets become unstable (pulsating), and their directionality is less pronounced. With the weakening of the jets, the condensation region shifts to the "accretion disk", where the "production" of non-baryonic matter is mainly carried out.

Nebulae taking part in its rotation, small and large celestial bodies, stars, and their clusters form visible "arms" of galaxies, which become "thinner" as they accelerate and sometimes stretch into very long filaments (Figure 6). Such threads penetrate the entire visible part of the Universe (Figure 9), which is the reason to call them the "web of the Universe". Its presence testifies to the prevalence in space of the phenomenon of substance overflow from one star or galaxy to another. This overflow of broadcasting is especially clear in "close systems of double stars or galaxies" (Figure 10). In this figure, the feature of this process is very clearly manifested, consisting in the invariability of the position of the centers of star clusters, while the peripheral layers move with acceleration. This confirms the existence of gravitational equilibrium, since $\nabla \rho=0$ at their centers of clusters. Equilibrium is violated only for the peripheral layers of these galaxies, where отлич $\rho$ are nonzero. They also experience gravitation, in which one star or galaxy, as it were, "strips" another. At the same time, it also follows from Fig. 10 that it is not necessarily the smallest of the galaxies that "undress": everything depends on the spontaneously arising density gradient of matter in a particular region of space, as it follows from the bipolar law of gravity (1). This law also easily explains why a significant part of the baryonic matter formed in the core of a spiral galaxy is ejected from its center in the direction of the rotation axis. The answer is that it is in this direction that the gradient of its density $\nabla \rho$, and, consequently, the gravitational force are minimal. Explains the bipolar law (1) and the concentric arrangement of galaxy clusters at a certain distance from the central cluster, discovered by NASA when building a three-dimensional map of the Universe. ${ }^{7}$ According to Newton's law, such an arrangement of clusters of stars is impossible due to their mutual attraction. However, according to the photograph in Figure 5, it really exists and is explained by the presence of gravitational equilibrium in the antinodes of star clusters, where $\nabla \rho=0$.

The proposed explanation of several observed phenomena confirms the validity of the energy-dynamic theory of strong gravity based on the law (1). This scenario makes it possible to distinguish the sequence of individual stages in the evolution of various regions of the Universe and to substantiate the circulation of matter in it. Therefore, it can become the basis of the energy-dynamic theory of its evolution. ${ }^{12}$

Along with this, there is a great doubt about the validity of the conclusions drawn based on general relativity. It becomes obvious that supermassive objects observed at the centers of most galaxies due to the absence of their glow are not always BHs predicted by general relativity. The nuclei of galaxies, which, like voids, are free of baryonic matter, can also be supermassive. It is they who, as they become denser, become "factories of stars", while BHs are only their "gravediggers". That is why the Swedish Academy of Sciences is right in awarding the prize to R. Hantsel and A. Ghez for "the discovery of a supermassive object in the center of our Galaxy." Thus, she left a chance that this object may not be a "black hole", especially since the "Schwarzschild sphere" is only a part of it.

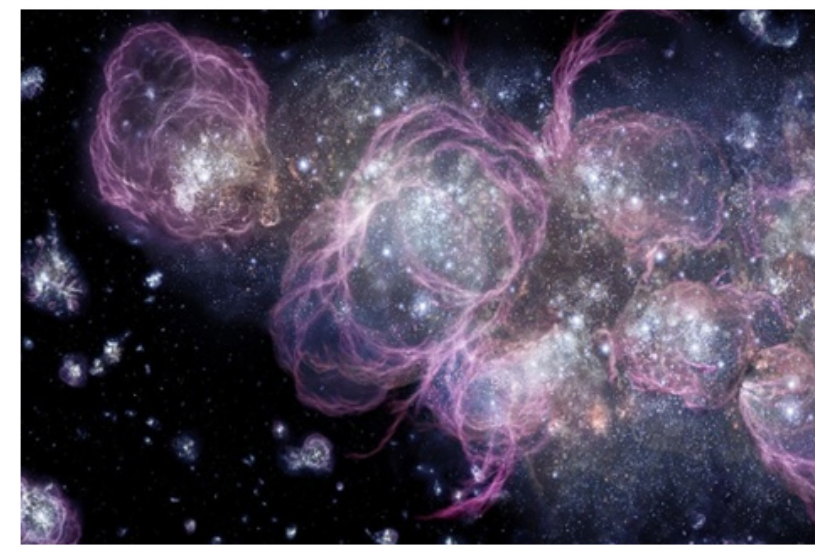

Figure 9 Filamentous structure formed by accretion arms.

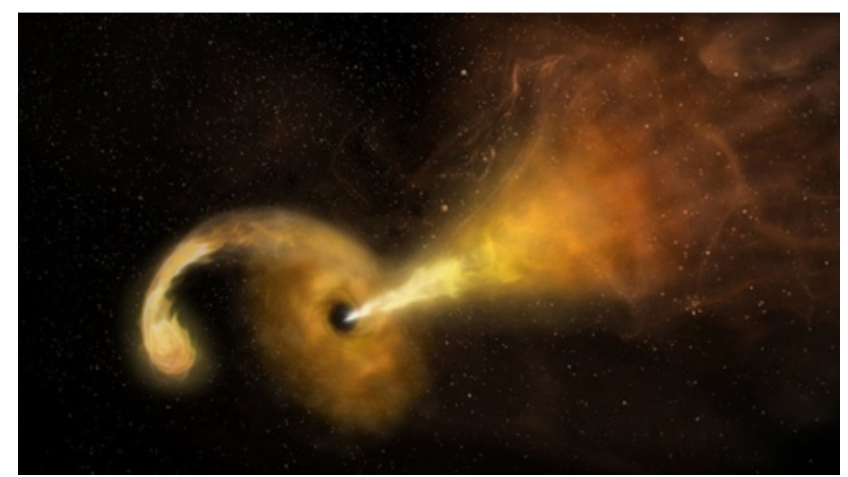

Figure 10 Overflow of matter to a galaxy with a lower mass.

However, this cannot be said about the R. Penrose Prize, whose contribution is assessed by the Nobel Committee as "the discovery that the formation of a black hole would be a reliable prediction of general relativity". The equations of this theory describe the homogeneous Universe "as a whole" without considering the opposite direction of the processes in its separate areas and therefore cannot correspond to the real situation. However, even without taking this into account, the formulation seems illegitimate, since the will of Alfred Nobel clearly provided for a prize for achievements that facilitate the life of mankind, and not for successes in the field of mathematical physics. In this regard, the example of Stephen Hawking, who was repeatedly nominated for the Nobel Prize, but did not receive it due to the impossibility of confirming his theory of "evaporating black holes", either by existing or possible in the future means of observation due to the infinity of the Universe, is very indicative.

\section{Acknowledgments}

None.

\section{Conflicts of interest}

Author declares that there is no conflict of interest. 


\section{References}

1. https://www.nobelprize.org/press-room/

2. Labeyrie A. Attainment of Diffraction Limited Resolution in Large Telescopes by Fourier Analysing Speckle Patterns in Star lmages. Astrophysics. 1970;6:85-87.

3. Linnik VP. On the fundamental possibility of reducing the influence of the atmosphere on the image of a star [In Russian]. Optics and Spectroscopy. 1957;4(4):401-402.

4. Duffner R.W. The Adaptive Optics Revolution: A History. University of New Mexico Press: New Mexico; 2009.

5. Penrose R. Collected Works: Six Volume Set. Oxford University Press; 2010.

6. Einstein A. Stationary system with spherical symmetry consisting of many gravitating masses. Annals of Mathematics (ser. 2). 1939;40(4):922-936.
7. Eisenstein DJ, Weinberg DH, Agol E, et al. SDSS-III: Massive Spectroscopic Surveys of the Distant Universe, the Milky Way Galaxy, and Extra-Solar Planetary Systems. Astron J. 2011;142:72.

8. Etkin V. Energodynamics (Thermodynamic Fundamentals of Synergetics). NewYork; 2011. 480 p.

9. Etkin V. Gravitational repulsive forces and evolution of the universe. Journal of Applied Physics (IOSR-JAP). 2016;8(6):43-49.

10. Etkin VA. Energodynamic theory of gravitation. Aeronautics and Aerospace Open Access Journal. 2019;3(1):40-44.

11. Eisenstein DJ, Zehavi I, Hogg DW, et al. Detection of the Baryon Acoustic Peak in the Large-Scale Correlation Function of SDSS Luminous Red Galaxies. The Astrophysical Journal. 2005;633(2):560-564.

12. Etkin V. On the Dialectic Unity of Evolution and Involution. Global Journal of Science Frontier Research: A Physics and Space Science. 2020;20(10):9-16. 